in ALBARELLO Luc, BARBIER Jean-Marie, BOURGEOIS Etienne, DURAND Marc (Dir.), 2013, Expérience, Activité, Apprentissage (111-133). PUF, Coll. Formation et Pratiques Professionnelles.

\title{
La sensibilité à, organisatrice de l'expérience vécue
}

Michel Récopé, Géraldine Rix-Lièvre, Hélène Fache, Simon Boyer

Michel.Recope@univ-bpclermont.fr

Clermont Université, Université Blaise Pascal, Laboratoire ACTé (EA 4281).

UFR STAPS, BP 104, 63172 Aubière cedex.

Cette contribution trouve sa raison dans nos insatisfactions de formateurs confrontés à l'importante variabilité interindividuelle de la «présence active ${ }^{1}$ des pratiquants et de leurs apprentissages : au volley-ball, certains progressent rapidement, d'autres quasiment pas. Les critères classiquement avancés (qualités physiques, volume de pratique antérieure, adresse spécifique, motivation à pratiquer) ne rendent pas compte de ces différentiels de "présence active » et d'apprentissage repérables dans les divers cadres de pratique (enseignement scolaire obligatoire et optionnel, secteur associatif). Cette insatisfaction éthique a généré une question de recherche visant une formation plus pertinente. Nos propos se limiteront au rapport qu'entretiennent en acte les joueurs avec le jeu et les situations du jeu. Le qualificatif de «pratiquants » souligne la restriction à ce seul aspect d'une activité assurément plus complexe.

La préoccupation permanente de formation et l'évolution du travail de terrain nous ont fait éviter toute école doctrinale pour retenir une position hybride: une anthropologie cognitive non mentaliste empruntant à des philosophies de la vie formant à nos yeux un ensemble d'orientations composites mais compatibles (Canguilhem, 2007 ; Jonas, 2001 ; Weber et Varela, 2002 ; Di Paolo, 2005 ; Barbaras, 2008); une éthologie phénoménologique des pratiques humaines ordinaires, perspective descriptive et comparative des actes inspirée de Thinès (1980), s'intéressant aux relations signifiantes avec le monde naturel spécifique (Umwelt). L'ensemble du propos s'inscrit dans l'approche énactive, une phénoménologie qui s'efforce de saisir l'expérience humaine pour remédier à l'absence de sens commun dans la définition de la cognition (Varela, 1989).

Le paradigme de l'énaction ${ }^{2}$ considère qu'il y a expérience dès qu'un organisme vivant constitue une unité intégrative s'incarnant dans un univers cognitif complexe et formant un centre de perspective et d'activité particulier sur le monde. Pour Varela (1998), cette expérience est une forme de conscience sans réflexion qui constitue un énorme background. C'est ce vécu in situ et in $a c t u$, qu'il distingue de l'expérience réflexive ou expérience de se référer à son propre vécu, que nous nous attacherons à décrire. Notre objectif est ambitieux car il vise les fondements inaccessibles du vécu : d'où vient que tel individu ait tel vécu, pourquoi tel phénomène apparaitil à tel individu ? Pour nous, l'expérience humaine est un processus historique indissociablement vital, culturel et individué conduisant en permanence à faire l'épreuve de soi en faisant l'épreuve du monde.

Au sein de ce paradigme, la cognition n'est pas la représentation plus ou moins adéquate d'un monde de propriétés prédonnées, mais l'avènement conjoint d'un monde et d'un esprit à partir de l'histoire des diverses actions qu'accomplit un être dans le monde (Varela, Thompson et Rosch, 1993). C'est une histoire de couplage structurel renvoyant à une logique de viabilité qui fait émerger un monde sensible (Varela, 1998). Les événements informationnels sont littéralement in-formati, c'est-à-dire formés au sein de ce couplage (Varela, 1989). Ce sont les schèmes de l'expérience corporelle et les structures préconceptuelles de notre sensibilité (à savoir notre mode de perception, notre manière de nous orienter et d'interagir avec d'autres

\footnotetext{
${ }^{1}$ Cette expression se veut, en première approximation, fidèle au sens commun désignant un dynamisme repérable par un observateur extérieur.

${ }^{2}$ Pour une présentation actualisée, on peut se reporter à Penelaud (2010).
} 
in ALBARELLO Luc, BARBIER Jean-Marie, BOURGEOIS Etienne, DURAND Marc (Dir.), 2013, Expérience, Activité, Apprentissage (111-133). PUF, Coll. Formation et Pratiques Professionnelles.

objets, événements ou personnes) qui nous permettent de posséder un monde (Varela et al., 1993).

Le pendant de cette approche de la cognition sur le plan de l'évolution des formes vivantes est la dérive naturelle, qui embrasse la temporalité des histoires vécues des espèces, des cultures et des individus (Varela et al, Ibid., 249-289). Elle conteste la conception adaptationniste et prescriptive de l'évolution, qui postule l'existence de solutions adaptatives plus ou moins optimales référées à des contraintes prédonnées et qui hiérarchise différents degrés adaptatifs. La conception de la dérive naturelle est post-darwinienne, elle postule une créativité adaptative débouchant sur une multiplicité de voies évolutives, seulement possibles parce que satisfaisant l'exigence suprême de viabilité. Elle considère qu'il y a émergence de différents modes adaptatifs indissociables d'une diversité d'Umwelts, c'est-à-dire de mondes phénoménaux incommensurables car non superposables les uns aux autres.

En conséquence, l'attention n'est portée ni sur le sujet ni sur l'objet, considérés comme des substances initialement co-présentes, mais sur la relation dynamique, circulaire, bidirectionnelle qui les lie, dans et par laquelle ils se spécifient conjointement (Sebbah, 2004). L'ontologie et l'épistémologie de l'énaction sont donc relationnelles (Goddard, 2003 ; Penelaud, 2010). Nous cherchons, à partir d'un terrain, à appréhender et à spécifier cette relation, que nous interpréterons comme sensibilité à.

\section{Présence active et régularités}

Le vécu comme l'activité sont toujours singuliers et contingents et la manière dont l'acteur peut les documenter est spécifiquement adressée. Ils peuvent cependant être approchés par le chercheur au moyen d'une schématisation en vertu du principe « quel que soit le type de système autonome que nous étudions, nous ne pouvons l'aborder qu'à partir de certaines régularités de son comportement, qui sont intéressantes pour nous, observateurs extérieurs, parce que nous avons un accès conjoint au fonctionnement du système et à ses interactions » (Varela, 1989, 10). Nos études (Fache, 2011 ; Récopé, Fache, Fiard, 2011) ont repéré différentes régularités, relatives aux : comportements in situ; situations dans lesquelles ils sont manifestés ; ressentis et significations exprimés a posteriori lors d'entretiens d'autoconfrontation à propos de ces comportements et situations. La mise en perspective de ces régularités constitue les matériaux supports de nos interprétations.

Les schématisations ainsi opérées ont abouti à la caractérisation de trois populations : trois manières d'être pratiquant, de vivre les situations du jeu de volley-ball. En effet, chaque population est repérable par sa surface adaptative ou son domaine d'interaction typique, deux expressions renvoyant pour nous au monde propre (Umwelt). La première est avancée par Cellérier (1992) : surface adaptative et surface évaluative sont indissociables, elles désignent les dimensions qualitatives caractérisant l'espace évaluatif qui détermine celles du milieu du système, soit sa niche écologique construite par les interactions précédentes. La seconde par Varela et al. (1993) : chaque mode d'adaptation définit une cohérence qui lui est propre et qui demeure tant qu'elle est viable pour le sujet dans le domaine d'existence de ses interactions. Une spécification qualitative et quantitative du domaine d'interaction de chaque population aboutit à sa caractérisation par une sensibilité propre.

D Pour les «peu actifs », pratiquer le volley-ball, c'est occuper une position sur le terrain et attendre, en attitude de repos (debout, détendus, bras ballants), que le ballon arrive à proximité pour le jouer. Une situation du jeu devient pertinente dès lors qu'ils constatent que le ballon est dirigé vers eux. Ils sont alors focalisés sur la réussite du bon placement des mains lors du contact avec le ballon. Ils ont incorporé une norme prévalente d'intervention sur le ballon, qui réfère à des formes strictement gestuelles de savoir-faire codifiés du volley-ball (la passe haute à deux 
in ALBARELLO Luc, BARBIER Jean-Marie, BOURGEOIS Etienne, DURAND Marc (Dir.), 2013, Expérience, Activité, Apprentissage (111-133). PUF, Coll. Formation et Pratiques Professionnelles.

mains et la manchette). Cette norme est d'origine culturelle, car de nombreux entraîneurs et formateurs abordent leur enseignement du volley-ball en valorisant l'importance accordée à l'exécution des bons gestes, ceux-ci ayant statut d'ingrédients fondamentaux de la pratique.

Leur domaine d'interaction est restreint : en effet, les circonstances du jeu font que le ballon arrive très rarement à proximité d'un joueur statiquement positionné en offrant des conditions d'intervention favorables. De plus, lorsqu'ils considèrent que le ballon est trop loin ou trop bas pour espérer réussir ce geste, ces pratiquants renoncent à intervenir. Leur activité semble dirigée vers l'exercice d'une opération au sens de Tassin (2007), laquelle implique un calcul des moyens ou de l'investissement au regard du résultat escompté.

Il est particulièrement difficile de remédier à leur «passivité » lors du processus enseignementapprentissage. De fait, lorsque la formation échoue à transformer leur "présence active », ils progressent très peu. Ils sont en effet prisonniers et victimes d'un paradoxe : la focalisation sur la phase finale du geste qu'ils cherchent à (re)produire occulte les préparations et ajustements conditionnant sa réussite. L'ensemble des fonctions afférentes à la défense de son camp et au soutien des partenaires leurs sont étrangères. On comprend mieux dès lors pourquoi ils sont les moins actifs et les moins performants.

Nous proposerons que l'activité et l'expérience vécue de ces pratiquants sont orientées par une sensibilité à la proximité du ballon (notée SPB).

D Pour les «moyennement actifs », pratiquer le volley-ball, c'est s'acquitter de sa part de travail et assumer des fonctions dévolues par répartition. Le bon déroulement du jeu de l'équipe repose sur une succession harmonieuse d'interventions individuelles. Pour ces pratiquants, les situations pertinentes du jeu sont celles dont l'évolution immédiate devrait normalement requérir leur intervention. C'est pourquoi se préparer, c'est pour eux s'apprêter à intervenir si leur responsabilité prédéterminée est engagée. Ils sont focalisés sur le résultat de leurs interventions, de leurs partenaires et adversaires en le rapportant au degré de difficulté du contexte. Ils ont incorporé une norme prévalente, d'origine culturelle, de devoir social: de nombreux formateurs présentent le volley-ball en insistant sur des critères d'organisation collective du jeu et sur les prescriptions afférentes qui prennent d'ailleurs souvent un caractère d'évidence pour de nombreux pratiquants.

Leur domaine d'interaction est caractérisé par une sorte d'intermittence sélective et conditionnelle : il y a les situations qui réclament une intervention de leur part, et celles qui ne la requièrent pas. De fait, lorsque leur responsabilité n'est pas en jeu, ils sont au repos, bien qu'attentifs aux déplacements du ballon. Lorsqu'ils constatent une intervention infructueuse d'un partenaire, ils estiment, avant d'intervenir, si un partenaire est mieux placé pour le faire et/ou les chances de réussite. Leur activité s'inscrit dans un rapport social au travail au sens de Tassin (2007), comme maillon d'une organisation collective instrumentale.

Nous proposerons que l'activité et l'expérience vécue de ces pratiquants sont orientées par une sensibilité aux responsabilités au sein de l'équipe (notée SRE).

D Pour ceux, peu nombreux, qui se révèlent «très actifs », pratiquer le volley-ball, c'est avant tout éviter la rupture de l'échange ${ }^{3}$ au détriment de leur équipe et la provoquer au détriment de l'adversaire. Le jeu est de bout en bout traversé par cet enjeu d'opposition. Leurs comportements attestent une proximité ${ }^{4}$ permanente à l'égard du ballon, mais s'ils s'approchent spatialement de

\footnotetext{
${ }^{3} \mathrm{Au}$ volley-ball, chaque « échange » débute par un service et se termine lorsque le ballon touche le sol ou lorsqu'un joueur commet une faute, il y a alors rupture de l'échange.

${ }^{4}$ Cette proximité doit être pensée au sens de Barbaras (2008, 319). Il s'agit d'une «proximité d'investissement » qui transcende le seul ordre spatio-temporel. Cette proximité engage l'existence toute entière et fait intervenir une dimension que l'on peut qualifier d'affective. Est proche ce vers quoi je suis tourné, ce dans quoi mon existence est engagée, bref ce dans quoi je suis investi.
} 
celui-ci, c'est pour s'éloigner existentiellement d'un événement : il faut absolument que le ballon ne tombe pas par terre. Ces pratiquants évaluent l'ensemble des situations du jeu en fonction de leur caractère plus ou moins menaçant au regard de cet enjeu, sachant que toute situation est comme destinée à devenir dangereuse. Cette préoccupation prend le pas sur toute considération liée aux aspects spatiaux, temporels, gestuels, organisationnels et d'intégrité corporelle du contexte d'intervention. Ils ne sont pas asservis à une stricte répartition des fonctions au sein du collectif et interviennent parfois dans l'urgence à la place d'un partenaire alors que jouer le ballon relève de la responsabilité de ce dernier.

Ils ont incorporé une norme prévalente s'avérant être celle, informelle, qui a fondé le sport volley-ball (Récopé, Fache, 2010) en tant que construit culturel. Nombreux sont les formateurs qui prescrivent une forte présence active des pratiquants sans travailler ses ressorts au cœur même de la formation; rares sont, en conséquence, les pratiquants qui la manifestent.

Leur domaine d'interaction est ample : redoutant la survenue d'un événement, ils agissent pour le pré-parer, pour s'en défendre, et sont naturellement intéressés par tous les moyens permettant d'y parvenir. Leur activité relève de l'action au sens de Tassin (2007) : un manifeste s'opposant à un état du monde, impulsé sans que soit envisagé le rapport entre investissement consenti et probabilités de réussite du résultat escompté.

Ces pratiquants les plus actifs se révèlent les plus performants et qui plus est ils progressent en cours de formation. Nous proposerons que leur activité et leur expérience vécue sont orientées par une sensibilité à l'enjeu de rupture de l'échange (notée SER).

L'examen des régularités met ainsi en évidence trois «présences actives » correspondant à trois populations de pratiquants caractérisés par leur rapport global au jeu. Les apprentissages spécifiques du volley, visés en formation, sont affectés par ce rapport. Nous avons cependant constaté qu'en cours de formation, certains pratiquants migrent de la première population vers la seconde et parfois vers la troisième. Les joueurs initialement peu actifs devenant plus actifs sont ceux qui progressent le plus (Récopé, 1996). Ces résultats assurant une première clarification du différentiel d'apprentissage nous ont incités à mieux comprendre pour mieux former.

\section{Sensibilité à et expérience vécue}

Les régularités repérables sont l'indice qu'au moment considéré de son histoire, chaque pratiquant vit des expériences et exhibe des comportements jamais identiques mais exprimant son identité et son monde de valeur ${ }^{5}$ par son couplage structurel avec son milieu. On ne peut mieux résumer :

«Only a small part of all dynamics in the environment enter as perturbations into the domain of relevance of the organism. All other possible interactions just fall outside of the possibilities of experience of the system [...] Contact with the world is thus always value, prefiguring in a prototypal form the qualities the world will unfold later according to this background. The perspective of a challenged and self-affirming organism lays a new grid over the world: a ubiquitous scale of value. To have a world for an organism thus first and foremost means to have value which it brings forth by the very process of its identity (Weber \& Varela, 2002, 118).

Les interprétations précédentes esquissent effectivement trois domaines de pertinence, indissociables de types d'interactions de nature et d'ampleur différentes dont le sujet peut faire l'expérience. Mais elles proposent en outre de spécifier comme sensibilité à, dans chaque domaine d'activité humaine, la relation que l'énaction met au cœur de sa conceptualisation. En

\footnotetext{
${ }^{5}$ Ceci est congruent avec toute approche dispositionnelle, telles celles valorisant les schèmes (Vergnaud, 2007) ou les dispositions (Durand, 2008), mais aussi les normes au sens de Canguilhem (2007) : on ne peut déterminer le normal par simple référence à une moyenne statistique mais par référence de l'individu à lui-même dans des situations successives ou variées.
} 
effet, cette approche «insiste sur l'interdépendance de l'esprit et du monde, tend à traiter leur relation [. . . ] comme si elle possédait une existence concrète indépendante » (Varela et al., 1993, 307). La sensibilité à concrétiserait ainsi, en réintroduisant le sens commun, l'ontologie et l'épistémologie relationnelles de l'énaction.

Si la vie est essentiellement relation, et si la relation implique une «transcendance », c'est-à-dire un aller-au-delà-de-soi qui ouvre à la possession d'un monde (Jonas, 2001), nous proposons de la caractériser comme sensibilité. Elle est le propre du vivant conçu comme totalité non autosuffisante dépendant de sa relation à l'extériorité. Elle est le fondement désirant, englobant les aspects cognitifs, affectifs et moteurs ${ }^{6}$, qui fait de la vie une activité dynamique s'opposant à l'inertie et à l'indifférence ${ }^{7}$. Selon Ribot, faculté désirante et sensibilité sont équivalentes : «la sensibilité, c'est la faculté de tendre ou de désirer et par suite d'éprouver du plaisir et de la douleur » (1896, 2). La sensibilité ouvre à l'appréciation, aux normes et valeurs, elle est ce pour quoi et par quoi il peut y avoir un monde indissociable d'un corps : elle est relation qualitative se déployant à la fois vers le monde et vers soi (Barbaras, 2003). Le désir est au cœur du sentir mais ne se manifeste pas à l'état pur, il s'exprime empiriquement et se révèle concrètement comme tendance, aspiration, mouvement vers se rapportant à des objets comme lieu ou condition de sa satisfaction possible (Barbaras, 2008) : il est désir de quelque chose. Ainsi, la sensibilité se manifeste toujours en tant que sensibilité à, relation actualisant concrètement et pratiquement un ensemble complexe de normes exprimant des orientations privilégiées portant l'individu vers certaines qualités d'objets, d'événements connus selon leur caractère de valeur (Canguilhem, 2007). L'appréhension du vécu d'un individu exige qu'on spécifie sa sensibilité individuée, c'est-à-dire la relation préférentielle qui ressort de son couplage structurel et circonscrit le domaine d'interaction et la classe des bonnes actions selon des critères de nécessité et de satisfaction propres (Maturana et Varela, 1994).

La sensibilité à prend pour nous le statut de valeur directrice dominant et organisant la totalité [activité-expérience-corps-monde], elle en est le sens pratique structurant (Récopé et al., 2011). Elle est la relation $\grave{a}^{8}$ la plus globale ${ }^{9}$ établissant ce qui importe, elle actualise in situ ce que le pratiquant tient pour pertinent dans les situations et ce qu'il y a lieu de faire ${ }^{10}$ durant le jeu. Elle détermine par là-même la téléologie intrinsèque (Jonas, 2001): si «ce qui fait l'organisme, c'est précisément que sa finalité sous forme de totalité lui est présente et est présente à toutes les parties » (Canguilhem, 2002, 120), c'est cette sensibilité à qui fait l'identité de l'organisme.

Elle résulte d'une incorporation individuée de normes/valeurs et de catégories dont l'origine est culturelle/collective. L'énaction pose qu'il n'y a «pas d'a priori, pas de syntaxe formelle régissant nos façons de catégoriser les éléments du monde. La catégorisation [...] émerge du couplage » (Penelaud, 2010, 9). Ainsi, le vécu de tout pratiquant est affecté par la médiation du formateur qui favorise souvent une sensibilité à constituant un obstacle aux apprentissages visés.

\section{Sensibilité, développement et apprentissage}

Une distinction de Maturana et Varela entre développement et apprentissage clarifiant le statut de la «structure » dans le couplage structurel nous semble cruciale pour la formation : «L'éventail des comportements possibles d'un organisme est déterminé par sa structure. Cette

\footnotetext{
${ }^{6}$ Pour Aristote, il n'y a qu'un seul principe moteur, la faculté désirante.

7 «Entre le vivant et son milieu, le rapport s'établit comme un débat où le vivant apporte ses normes propres d'appréciation des situations, où il domine le milieu et se l'accommode » (Canguilhem, 2003, 187).

${ }^{8}$ Par opposition à une relation entre des entités substantielles (Utaker, 2007)

9 «Une description globale resserre tous les phénomènes autour d'un centre unique... » (Foucault, 1969, 19). Pour Canguilhem (2003), tout milieu de vie suppose un centre absolu de référence.

${ }^{10}$ Le caractère normatif est patent puisque la norme fixe un cadre à l'agir : ce qui doit être (Durrive, 2010, 28).
} 
in ALBARELLO Luc, BARBIER Jean-Marie, BOURGEOIS Etienne, DURAND Marc (Dir.), 2013, Expérience, Activité, Apprentissage (111-133). PUF, Coll. Formation et Pratiques Professionnelles.

structure spécifie son domaine d'interaction » $(1994,164)$. L'ontogenèse est considérée comme «l'histoire individuelle des changements structuraux», alors que «l'apprentissage est l'expression d'un couplage structural qui veille à maintenir la compatibilité des interactions de l'organisme opérant et de son environnement » (ibid., 165).

Ces perspectives conduisent à distinguer deux types d'acquisition :

- le développement, conçu comme épisode qualitatif de changement structural, c'est-à-dire comme épisode de reconfiguration du domaine d'interaction et des catégorisations sous-jacentes, puisque l'organisme et son environnement co-adviennent simultanément (Penelaud, 2010). «Évoluer, veut alors dire : développer son rapport au monde» (Ibid., 6) soit l'énaction d'un nouveau monde. Nous considérons le développement comme une «acquisition » particulière faite dans et par la vie, assurant l'émergence d'une nouvelle sensibilité à, donc d'un nouvel état global : une reconfiguration ouvrant à une nouvelle unité de perspective et d'agentivité, donc à de nouvelles possibilités d'expérience et d'activité.

Notre inscription énactive, non adaptationniste, n'assure aucune différence épistémologique ni ontologique entre les différentes sensibilités à: il ne peut y avoir en soi de «meilleure » sensibilité à, puisqu'elles résultent toutes du processus de dérive naturelle.

- l'apprentissage, conçu comme la sous-classe des acquisitions opérées dans le cadre d'un même couplage structural. Il correspondrait ainsi à une amélioration d'ordre quantitatif des interactions actuelles, c'est-à-dire à l'acquisition de procédures permettant leur efficacité accrue. Si l'éventail des comportements possibles comme le domaine d'interaction d'un organisme sont contraints par la structure, alors les apprentissages possibles le sont également, dès lors qu'on accorde un poids phénoménologique au fait que toutes les autres interactions envisageables sont, au moment considéré, étrangères aux possibilités d'expérience (Weber \& Varela, 2002). Le constat qu'à un domaine d'interaction restreint (ample) correspondent des apprentissages restreints (amples) n'est alors pas étonnant, mais confirme la radicalité épistémologique et théorique de la perspective énactive. Dans la mesure où les sensibilités à sont solidaires chacune d'un domaine d'interaction particulier, plus ou moins ample, la nature et l'ampleur de ce qui est « apprenable » sont donc également par celles-ci circonscrites.

La distinction entre changement qualitatif global et changement quantitatif peut sembler hasardeuse: elle a pour nous le statut d'hypothèse interprétative des inégalités d'apprentissages dont nous constatons sur le terrain qu'elles se creusent généralement lors de la formation ${ }^{11}$. Elle ne consiste pas en un choix a priori, mais ressort a posteriori de nos études, suite :

- au repérage, après examen conjoint des matériaux, de trois modes différents de pratique viable nous semblant relever de trois sensibilités à ;

- au constat que l'évolution ne répond ni à une logique de «stades » (au sens d'un passage nécessaire par l'un des états avant d'accéder à un autre), ni à une logique d'expertise : des débutants peuvent d'emblée s'avérer SER, des joueurs de meilleur niveau se révèlent SRT ;

- au repérage de changements importants, d'ordre global, du mode de pratique chez certains joueurs : l'ancienne régularité comportementale laisse place à une nouvelle régularité.

\section{Une formation centrée sur la sensibilité, le développement et le vécu}

La sensibilité est donc la cible de notre formation, le plus tôt possible, pour tous les pratiquants : notre travail ayant été généré/alimenté par des questions éthiques liées à une visée professionnelle, nous récusons la césure chercheur/formateur ${ }^{12}$. Aussi assumons-nous d'être des

\footnotetext{
${ }^{11}$ Ce qui nous heurte, puisque que nous croyons que l'accroissement de l'inégalité des chances est contraire à l'idéal républicain.

${ }^{12}$ Il nous est difficile de séparer nos observations, nos questions et problématisations en périodes de formation et d'autres de recherche.
} 
manipulateurs suscitant des vécus compatibles avec les apprentissages spécifiques du volleyball. Nous sommes conscients d'être normalisateurs, en ce que nous visons à réduire l'écart ${ }^{13}$ entre la sensibilité à initiale (la norme/valeur et les conceptualisations en acte sous-jacentes) des pratiquants et celle qui nous paraît favoriser ces acquisitions. L'enjeu principal est donc de susciter l'avènement de la SER chez les formés, à partir de leur identité actuelle, pour «transformer » leur vécu en référence aux standards de performance.

Il s'agit donc de rompre avec les formations ayant cours. Nous observons qu'en dépit d'un « ordre de mobilisation générale » des pratiquants, la quasi-totalité des formateurs induisent en réalité, en raison des contenus qu'ils dispensent, une sensibilité à qui ne favorise pas une telle mobilisation. Les prescriptions exhortent de l'extérieur à bouger, anticiper, se livrer à fond, aller au ballon, être concentré sur le jeu, faire attention, se préparer, se déplacer tôt et rapidement, lire tôt les trajectoires du ballon, défendre tous les ballons, aller soutenir les partenaires, etc. Ces injonctions restent souvent lettre morte (y compris pour des joueurs de bon niveau) car elles échappent à une problématisation des conduites (Marsenach, 1991).

\section{Une coordination d'activités et de vécus}

Une modélisation des conduites peu à peu construite au sein de notre processus formation-recherche propose que l'activité et le vécu du pratiquant sont issus de la coordination de plusieurs registres. Non isolables, ces derniers constitueraient chacun un module perceptionaction établissant ses propres événements informationnels et assurant ses contributions motrices à l'activité d'ensemble. Une pluralité de vécus et d'activités caractériserait donc le tout complexe et organisé émergeant de ces registres interactifs. Cette proposition est compatible avec l'affirmation que la cognition opère au moyen d'un réseau consistant en niveaux multiples de sous-réseaux sensori-moteurs interconnectés (Varela et al., 1993), déjà envisagée par Reed (1982) : les actions ne sont pas des unités discrètes mais des relations imbriquées impliquant diverses boucles perceptivo-motrices mutuellement ajustables; les composants des actions sont eux-mêmes des actions ; lorsque plus d'un composant est impliqué dans une action (comme c'est presque toujours le cas), aucun d'eux n'apparaît individuellement de façon évidente.

Ce modèle envisage des processus fonctionnels et «leur rapport d'insertion dans la totalité indivisible d'un comportement individuel » (Canguilhem, 2007, 50) : les acquisitions opérées au sein d'un registre favorisent certaines acquisitions relevant d'autre(s) registre(s). Chaque pratiquant, en fonction de son histoire, possède des acquis plus ou moins importants dans l'un ou l'autre des registres : différencier l'enseignement présuppose alors l'identification du (ou des) registre(s) qui se révèle(nt) le(s) plus limitant(s) pour son action.

\section{Le registre de la sensibilité à : un corps sensible indissociable d'une mobilisation en situation.}

Il détermine la téléologie du pratiquant, donc ses raisons d'agir les plus globales. Correspondant à la source d'investissement de soi dans le monde ${ }^{14}$, il est susceptible d'un développement.

Cette mobilisation (Récopé, Lièvre, Rix-lièvre, 2010) s'exprime au sein de domaines d'interaction d'ampleur et de nature différentes. Elle réfère à des pratiquants plus ou moins concernés par les déplacements du ballon, intéressés à défendre, à anticiper, etc. Elle se concrétise par la fréquence relative de postures de repos et de garde, par la présence permanente ou l'absence: d'orientation face au ballon, de déplacements exploratoires, de courses vers le ballon sous formes de jaillissements instantanés, de chutes au sol, de manifestations

\footnotetext{
${ }^{13}$ Toute formation est normalisatrice. A nos yeux, une formation d'inspiration behavioriste vise une réduction d'écart entre les comportements actuels et les comportements attendus ; une formation d'inspiration cognitiviste vise une réduction d'écart entre les connaissances actuelles et la réalité objective du monde.

${ }^{14}$ Selon la belle expression suggérée par Pastré (communication personnelle).
} 
in ALBARELLO Luc, BARBIER Jean-Marie, BOURGEOIS Etienne, DURAND Marc (Dir.), 2013, Expérience, Activité, Apprentissage (111-133). PUF, Coll. Formation et Pratiques Professionnelles.

émotionnelles de frustration, etc. Ainsi, à l'inverse des SER, les pratiquants SPB demeurent statiques, en position de repos, debout, bras ballants aussi longtemps qu'ils n'ont pas constaté que le ballon arrive à leur proximité. Lorsque c'est le cas, ils mobilisent leurs bras pour le contacter, et déplacent seulement un appui pédestre à cette fin. Aucune partie de leur corps, autre que les appuis, ne touche jamais le sol, sauf chute involontaire, extrêmement rare.

Ce registre englobe toutes les acquisitions permettant de devenir plus sensible à l'enjeu de rupture de l'échange : l'intériorisation du volley comme sport saturé par un enjeu d'opposition ; l'intégration des différences de projet d'utilisation du ballon entre « jeu avec » (assurer une continuité de l'échange avec les partenaires) et «jeu contre » (rechercher au plus tôt la rupture de l'échange au détriment des adversaires); la distinction entre «balle cadeau » (ne provoquant pas de crise spatio-temporelle) et «balle méchante » (induisant une crise spatio-temporelle menaçant du danger de rupture et obligeant à s'employer); l'intention constante de se donner -et d'offrir aux partenaires- les meilleures conditions de contact et de visée et d'interdire aux adversaires de bonnes conditions de visée, voire la possibilité même de contacter le ballon.

Les acquisitions spécifiques de ce registre sont essentielles, car le développement de la SER s'accompagne d'une disponibilité corporelle, servie par une relation tonico-affective (Wallon, 1949) dont témoigne l'état de garde, et ouvre l'accès aux apprentissages relatifs aux autres registres. Tout est donc mis à profit pour créer un contexte de formation propice : présentation du jeu par une mise en scène de l'enjeu de rupture ; insistance «surjouée » sur le danger de rupture et la nécessité de se préserver des crises spatio-temporelles, sur le fait que «bien jouer », c'est les réduire ou les résorber pour soi et ses partenaires, les provoquer chez les adversaires ; formules «humoristiques » telles «balle au sol me désole, balle en vie me réjouis », «pas de crise, je me frise »; nombreux exercices de type « défi » lancés aux formés ou qu'ils se lancent.

Nos constats attestent que: quelques pratiquants seulement ont acquis cette sensibilité avant même l'entrée en formation; de nombreux pratiquants deviennent plus sensibles à l'enjeu de rupture de l'échange à l'issue d'une douzaine d'heures de formation, grâce au champ d'actions encouragées (Reed et Bril, 1996) à la fois instrumenté et artisanal ${ }^{15}$ que nous instaurons ; quelques pratiquants ne l'incorporent pas, ou peu, en dépit de nos efforts.

Ce registre ne suffit cependant pas pour agir efficacement en volley-ball et ne recouvre pas toutes les acquisitions requises. La contribution de trois autres registres, plus spécialisés, est nécessaire.

\section{De registre de la pertinence tactique : un corps de rationalité pratique s'efforçant de contextualiser efficacement ses actions}

La pertinence tactique réfère à la mise en œuvre de solutions permettant d'harmoniser les possibles personnels et le contexte actuel du jeu. Elle requiert des décisions d'intervention in situ des pratiquants reposant sur de nombreuses connaissances ayant le statut de ressources pour l'action. C'est nécessaire car les déplacements aériens du ballon étant permanents, les situations du jeu évoluent en temps réel. Sur ce plan encore, il y a une importante différence entre les pratiquants SPB et SER quant aux événements in-formati. Les premiers, caractérisés par un empan temporel et situationnel restreint, sont focalisés sur les moyens de bien placer les mains sur le ballon. Les ajustements pertinents consistent à bien "préparer les mains » juste avant le moment du contact; ils réclament avec insistance des indications sur le bon placement qui a, pour eux, valeur de moyen-clé à apprendre. Cette focalisation exclusive fait qu'ils sont en attente passive, non concernés par les déplacements du ballon tant que ce dernier n'est pas dirigé vers eux. Leur réaction tardive est souvent rapportée à de prétendues difficultés de lecture/d'appréciation des trajectoires du ballon, mais selon nos interprétations, la raison

\footnotetext{
${ }^{15}$ Se rapporter à Récopé, Barbier, Rix et Jaujou (2008) pour des précisions sur la formation des débutants.
} 
in ALBARELLO Luc, BARBIER Jean-Marie, BOURGEOIS Etienne, DURAND Marc (Dir.), 2013, Expérience, Activité, Apprentissage (111-133). PUF, Coll. Formation et Pratiques Professionnelles.

essentielle est ailleurs : en effet, s'intéresser tôt à la trajectoire du ballon n'est pertinent que si on est intéressé à prévoir son lieu de destination.

Les seconds sont caractérisés par un empan temporel et situationnel large, puisqu'ils anticipent l'évolution des situations du jeu comme possiblement dangereuse. Leur préoccupation est de pré-parer un événement qu'ils ne peuvent pas sentir. Cherchant à l'éviter, ils sont enclins à la réflexivité sur les conditions situationnelles et réglementaires, sur ce qu'il aurait fallu faire et ce qu'il faudrait faire à l'avenir pour ne plus le vivre. Par exemple, l'un d'entre eux, joueur de niveau mondial, éprouve le besoin de bien connaître le jeu des meilleurs serveurs, ceux qu'il reconnaît comme pouvant le mettre en défaut, avant de les affronter. Il demande des montages de films actualisés pour repérer leurs habitudes et se livre à une recherche minutieuse de régularités (entre leur lancer de balle et la direction que prend leur service ; le service «sûr » qu'ils utilisent lors des fins de set ou de match ; leur type de service le plus fréquent). Ceci facilite sa prévision et offre en outre des solutions de perturbation/dissuasion de ces serveurs, par un jeu de placements adéquats au moment même où celui-ci s'apprête à servir (indications d'ajustement à destination de ses partenaires pour surcharger la zone de destination probable du service).

Ce registre concerne tous les apprentissages permettant de réguler ses actions pour mieux les contextualiser grâce à des enseignements tirés de son histoire. Ces apprentissages répondent en particulier à la volonté de ne pas faire des «cadeaux» à l'adversaire et consistent en l'établissement de rapports entre conditions situationnelles, modalités d'intervention et degré de réussite, rapports progressivement étendus aux possibilités personnelles (points forts/points faibles), à celles de son équipe et de l'équipe adverse.

De registre de la visée par déviation : un corps instrument de la précision des interventions. L'efficacité des interventions dépend en volley-ball de la précision des trajectoires produites. Il est indispensable d'exercer un contrôle gestuel suffisamment long sur le ballon pour produire les forces lui imposant la trajectoire souhaitée, ce qui suppose une intention de visée ainsi que la préparation de celle-ci avant même d'avoir contacté le ballon. Les apprentissages relevant de ce registre réclament de nombreuses répétitions dans des conditions de placement corporel favorisant la visée.

Le statisme des pratiquants SPB ne permet pas de satisfaire ces conditions, du fait de l'absence de déplacement exploratoire avant le contact du ballon. De fait, ils touchent peu de ballons, sont en général loin de lui et réagissent trop tardivement pour pouvoir le contrôler (ils projettent les bras au moment du contact). Tant qu'ils restent stimulés par l'arrivée du ballon, ils sont condamnés à des réactions tardives et explosives et ne peuvent progresser que sur des aspects minimes liés à la réalisation du contact. En revanche, les pratiquants SER, beaucoup plus mobiles, se déplacent tôt et vite pour éviter/compenser les crises spatio-temporelles. Constamment focalisés sur les déplacements du ballon, toujours préoccupés de s'en approcher, ils cherchent à se placer avant lui à l'endroit où il va arriver. Mieux et plus tôt placés, en mesure de toucher plus souvent le ballon, ils peuvent mettre en œuvre et réguler les solutions gestuelles de visée par déviation qui sont les apprentissages spécifiques de ce registre : création d'un temps de repérage et de visée, incorporation de zones de visée, allongement des membres supérieurs appliquant des forces directionnelles sur le ballon, etc. Ces apprentissages reposent sur la construction progressive d'un bon compromis entre les exigences mécaniques de la déviation du ballon, les conditions spatio-temporelles de la situation et les caractéristiques anatomiques du corps humain. Ceci présuppose un pratiquant correctement placé : or les formateurs, même s'ils admettent que la qualité du placement détermine la qualité de l'intervention, ne s'intéressent pas assez aux raisons d'agir qui fondent la nécessité de bien se placer avant de jouer le ballon.

De registre de l'équilibration spécifique : un corps postural condition de l'efficacité des déplacements et de la visée 
Ce registre englobe l'ensemble des apprentissages spécifiques autorisant les régulations équilibratrices nécessaires aux déplacements efficaces et aux régulations des postures indispensables à toute visée. Trop souvent minoré dans la formation, méconnu car il constitue l'arrière-plan permanent mais discret des interventions, il implique l'ensemble du corps. Sa fonction est d'autoriser des appuis rapides et efficaces, d'assurer la stabilisation des placements, de libérer les membres supérieurs pour exercer la visée, autant d'aspects plus ou moins favorisés ou contrariés par l'histoire de chaque pratiquant, du rapport d'équilibration au sol qu'il a auparavant construit. Les apprentissages visent un corps jouant avec les lois de la gravité, un corps se soumettant aux exigences de l'intervention et de la visée, disponible pour s'ajuster aux circonstances et pour réguler ses différents segments (par dissociations et coordinations) dans des conditions jamais identiques.

Les pratiquants SPB, vu leur passivité, sollicitent très peu ces diverses fonctions. Leurs déplacements limités, leurs ajustements d'appuis ne survenant que pour compenser un déséquilibre produit par la projection des bras sur le ballon, leur renoncement à jouer le ballon lorsqu'ils estiment ne pas pouvoir réussir le bon geste, font qu'ils ne s'engagent pas dans des postures inédites. Ils n'explorent pas de nouvelles sensations équilibratrices, ne mettent pas en péril leurs schémas posturaux habituels et n'accèdent donc pas aux équilibrations spécifiques du volley-ball. En revanche, les pratiquants SER, en raison de leur mobilisation intense, de leurs nombreuses tentatives opérées à partir d'une position jambes fléchies et sur la base d'un tonus postural élevé (deux composantes de leur état de garde), s'aventurent dans des solutions équilibratrices inédites, souvent jusqu'à la chute. Ils explorent spontanément de nouvelles formes d'appuis et de sensations, remaniant ainsi leurs schémas posturaux initiaux vers ceux requis par la pratique du volley-ball.

«Sans qu'ils soient séparés ou autonomisés, les territoires sont différenciés et jouissent d'une relative autonomie. La pénétration réciproque de toutes les parties supposées est le propre de tout organisme » (Dagognet, 1997, 110); de même, le monde se dresse sur le fond d'une série continue d'événements signifiants reliés entre eux (Varela et al., 1993). Le modèle de la coordination des registres nous paraît rendre compte d'une unité intégrative assurant un centre de perspective et d'activité sur le monde (Di Paolo, 2005) car il envisage l'organisation de l'activité et du vécu des pratiquants comme la résultante de l'interaction d'unités modulaires mais intégrées (Varela, 1998). Enfin, et de manière non paradoxale, l'orientation de l'activité et du vécu est assurée par le premier registre, celui de la sensibilité à, instaurant une activité qui est orientée sans être consciente, dont ne rendent compte ni le mécanisme ni l'intellectualisme classiques (Barbaras, 2008).

\section{Conclusion}

La variété de la sensibilité à, que nous définissons comme le sens structurant la totalité [activité-expérience-corps-monde], rend compte des différentiels de présence active et d'apprentissage observés chez les pratiquants de volley-ball. Les diverses sensibilités à que nous pensons avoir identifiées instaurent autant d'identités de pratiquants vivant leur expérience au sein de domaines d'interactions mais aussi d'acquisitions, d'ampleur et de nature différentes. Nous avons suggéré l'urgence d'une formation résolument centrée sur la valorisation de la sensibilité à la plus compatible avec les apprentissages visés dans un domaine d'activité. Cette proposition nous paraît radicaliser les fondements de l'approche énactive. Elle nous paraît en outre compatible avec les analyses de Pastré (2005): nous faisons l'hypothèse que le développement de la sensibilité à constitue un épisode de rupture, soit une modalité de genèse identitaire emportant les autres genèses (instrumentales, conceptuelles, opératives) passées en revue par cet auteur. L'émergence par trans-formation de la sensibilité à engloberait donc une 
in ALBARELLO Luc, BARBIER Jean-Marie, BOURGEOIS Etienne, DURAND Marc (Dir.), 2013, Expérience, Activité, Apprentissage (111-133). PUF, Coll. Formation et Pratiques Professionnelles.

reconfiguration de l'unité complexe [activité-expérience-corps-monde], processus qu'il nous faudra désormais appréhender.

Mais tout ceci n'est au fond qu'un compte rendu de notre expérience réflexive, en tant que formateurs/chercheurs dont la sensibilité à l'inégalité d'apprentissage a été et reste en souffrance.

Aristote (2005). De l'âme. Traduction P. Thillet. Paris : Gallimard.

Barbaras, R. (2003). Vie et intentionnalité. Recherches phénoménologiques. Paris : Vrin.

Barbaras, R. (2008). Introduction à une phénoménologie de la vie. Paris : Vrin.

Canguilhem, G. (2002). Le problème des régulations dans l'organisme et dans la société. Ecrits sur la médecine. Paris : Seuil.

Canguilhem, G. (2007). Le Normal et le Pathologique. Paris : PUF (1966).

Cellérier, G. (1992). Les fondements du constructivisme psychologique. In B. Inhelder \& G.

Cellérier (Eds.), Le cheminement des découvertes de l'enfant (213-302). Neuchâtel : Delachaux et Niestlé.

Dagognet, F. (1997). Georges Canguilhem, Philosophe de la vie. Le Plessis-Robinson : Institut Synthélabo.

Di Paolo, E. A. (2005). Autopoiesis, adaptivity, teleology, agency. Phenomenology and the Cognitive Sciences, 4, 429-452.

Durand, M. (2008). Un programme de recherche technologique en formation des adultes. Éducation et Didactique, 2(2), 69-93.

Durrive, L. (2010). L'activité humaine, à la fois intellectuelle et vitale. Travail et Apprentissages, 6, 25-45.

Fache, H. (2011). L'expérience corporelle des pratiquants de Volley-ball en situation. Phénomènes et structure des apparaissants. Sarrebrück : Éditions Universitaires Européennes.

Foucault, M. (1969). L'archéologie du savoir. Paris : Gallimard.

Goddard, J.-C. (2003). Autonomie, réduction et réflexivité. Intellectica, 1-2(36-37), 205-225.

Jonas, H. (2001). Le phénomène de la vie. Bruxelles, de Boeck (1966).

Marsenach, J. (1991). Education Physique et Sportive : Quel enseignement? Paris : INRP.

Maturana, H. R., Varela, F. J. (1994). L'arbre de la connaissance. Paris : Addison-Wesley.

Penelaud, O. (2010). Le paradigme de l'énaction aujourd'hui. Plastir, 1, 18.

Pastré, P. (2005). Genèse et Identité. In P. Rabardel \& P. Pastré (Eds.), Modèles du sujet pour la conception (231-259). Toulouse : Octarès Editions.

Récopé, M. (1996). Statut et fonction du schème du duel dans l'organisation de l'action motrice d'opposition : le cas du volley-ball. Thèse de Psychologie, Université Paris 5.

Récopé, M., Fache, H. (2010). La sensibilité incorporée des volleyeurs les plus «actifs ». In A. Berthoz \& B. Andrieu (Eds.), Le corps en acte (83-108). Nancy : PU de Nancy.

Récopé, M., Fache, H., Fiard, J. (2011). Sensibilité, conceptualisation et totalité [activitéexpérience-corps-monde], Travail et Apprentissages, 7, 11-32.

Récopé, M., Lièvre, P., Rix-Lièvre, G. (2010). The commitment of polar expedition members to a project. Project Management Journal, 41(3), 45-56.

Récopé, M., Barbier, D., Rix, G., Jaujou, C. (2008). Volley-ball. In M. Récopé \& B. Boda (Eds.), Raisons d'agir, raisons d'apprendre. Dossiers EPS n76 (25-46). Paris : Editions EP.S.

Reed, E. S. (1982). An Outline of a Theory of Action Systems. Journal of Motor Behavior, 14, 2, 98-134.

Reed, E. S., Bril, B. (1996). The primacy of action in development. In M. L. Latash \& M. T. Turvey (Eds.), Dexterity and its development (431-451). Hillsdale, N J: Lawrence Erlbaum.

Schwartz, Y., Durrive, L (2009). L'activité en Dialogues. Toulouse : Octarès. 
in ALBARELLO Luc, BARBIER Jean-Marie, BOURGEOIS Etienne, DURAND Marc (Dir.), 2013, Expérience, Activité, Apprentissage (111-133). PUF, Coll. Formation et Pratiques Professionnelles.

Sebbah, F.-D. (2004). L'usage de la méthode phénoménologique dans le paradigme de l'énaction. Intellectica, 2(39), 169-188.

Tassin, E. (2007). Entretien avec Etienne Tassin. Le Philosophoire, 29, 13-40.

Thinès, G. (1980). Phénoménologie et sciences du comportement. Liège : Mardaga.

Utaker, A. (2007). Canguilhem et Wittgenstein. In Jean-François Braunstein (Ed.), Canguilhem, Histoire des sciences et politique du vivant (143-161). Paris : PUF.

Varela, F. (1989). Autonomie et connaissance. Paris : Seuil.

Varela, F. (1998). Entretien avec Francisco Varela. La Recherche, 308, 109-112.

Varela, F., Thompson, E., Rosch, E. (1993). L'inscription corporelle de l'esprit. Paris : Seuil.

Vergnaud, G. (2007). Héritages. In M. Merri (Ed.), Activité humaine et conceptualisation (2737). Toulouse : PU du Mirail.

Wallon, H. (1949). Les origines du caractère chez l'enfant. Paris : PUF.

Weber, A., Varela, F. (2002). Life after Kant: Natural purposes and the autopoietic foundations of biological individuality. Phenomenology and the Cognitive Sciences, 1, 97-125. 